\title{
An Overview of Pathogenesis, Complications and Management of Rheumatic Heart Disease
}

\author{
Leong Tung Ong* \\ Faculty of Medicine, University of Malaya, Kuala Lumpur, Malaysia \\ *Corresponding author: Leong Tung Ong, Faculty of Medicine, University of Malaya, Kuala Lumpur, Malaysia
}

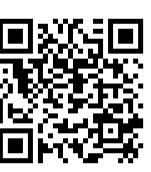

\section{ARTICLE INFO}

Received: 幽 July 16, 2020

Published: 蔧 August 07, 2020

Citation: Leong Tung Ong. An Overview of Pathogenesis, Complications and Management of Rheumatic Heart Disease. Biomed J Sci \& Tech Res 29(3)-2020. BJSTR. MS.ID.004793.
ABSTRACT

Rheumatic heart disease (RHD) is damage of the heart valves due to acute rheumatic fever (ARF) which results from the body's autoimmune response to Streptococcus pyogenes (group A Streptococcus bacteria) infection which is a throat infection. Acute rheumatic fever is one of the most important causes of cardiovascular morbidity and mortality in the developing countries although the incidence has decreased in North America, Europe and developed countries over the past 4 to 6 decades. The clinical symptoms of ARF which occurs approximately two to four weeks after $S$. pyogenes infection. The most common clinical manifestations of ARF are carditis and arthritis, followed by chorea and then subcutaneous nodules and erythema marginatum.

Keywords: Rheumatic Heart Disease; Acute Rheumatic Fever; Pathogenesis;Complications;Management

\section{Mini Review}

Rheumatic heart disease (RHD) is damage of the heart valves due to acute rheumatic fever (ARF) which results from the body's autoimmune response to Streptococcus pyogenes (group A Streptococcus bacteria) infection which is a throat infection. Acute rheumatic fever is one of the most important causes of cardiovascular morbidity and mortality in the developing countries although the incidence has decreased in North America, Europe and other developed countries over the past 4 to 6 decades [1]. The clinical symptoms of ARF which occurs approximately two to four weeks after $S$. pyogenes infection [2]. The most common clinical manifestations of ARF are carditis and arthritis, followed by chorea and then subcutaneous nodules and erythema marginatum [1].

\section{Pathogenesis}

Autoimmune reactions in ARF and RHD are caused by molecular mimicry between Streptococcus pyogenes antigens and human proteins which is a type II hypersensitivity [3]. During streptococcal infection, antigen presenting cells such as dendritic cells and B cells present the streptococcal antigens to naïve CD4+ $\mathrm{T}$ cell and lead to activation to helper $\mathrm{T}$ cell. $\mathrm{B}$ cells undergo receptor-mediated endocytosis of antigen and present as class II major histocompatibility complex which is then recognized by activated helper T cells to activate B cells. Activated B cells in the germinal centers differentiate into plasma cells which then produce antibodies which are part of a normal humoral response against S. pyogenes bacteria. However, the antibodies produced cross react with antigens in heart tissue or joints which lead to neutrophil and macrophage activation and inflammation.

Molecular mimicry in ARF occurs due to the alpha-helical protein structures found in $\mathrm{M}$ protein and N-acetyl-beta-Dglucosamine which is the carbohydrate antigen of $S$. pyogenes cross react with cardiac myosin, myocardium and valves.4 Binding of cross-reactive antibodies causes upregulation of vascular cell adhesion molecule 1 which results in adherence of CD4+ T cells to the endothelium and subsequently the cells enter the valve [2]. Inflammation occurs when T-cells release $\gamma$-IFN due to TH1 response which will lead to neovascularization and further T-cells recruitment [2]. $\mathrm{T}$ cells infiltrate the heart valves and result in scarring due to inflammation of the endothelium surrounding the valve [4]. Besides that, autoantibodies against type I collagen are also produced can be caused by aggregation of collagen by certain streptococcal serotypes or damaged valve which release collagen in rheumatic heart disease [4]. 
Genetic predisposition plays an important role in increasing susceptibility of RHD but the exact underlying mechanisms are not well understood. ARF susceptibility is most likely due to polymorphisms in several gens coding for immune proteins which are genetic associations related to class II human leukocyte (HLA) molecules [2]. HLA class II DR and DQ loci in chromosome 6 are often associated with the risk of acquiring RHD where certain HLA alleles or genotypes can be associated with increased risk or protection relating to ARF and the development of RHD [5]. This is because HLA alleles involve in regulating the immune response to infections by presenting antigens to T-cells receptors, autoimmune response by binding and presenting autoantigens, regulating selection of $\mathrm{T}$ cells and can serve as target autoantigens at the same time [5].

\section{Complications}

RHD damages the heart valves after group A streptococcal infection during episodes of ARF which will lead to valvular heart disease. The patient may or may not develop RHD after episodes of ARF which depends on the presence, type or severity of valvular disease.6 RHD can lead to congestive heart failure due to the involvement of mitral valve which results in mitral stenosis (MS) and mitral regurgitation (MR), occasionally aortic valve can be involved [2]. RHD causes damage of mitral subvalvular apparatus which causes mild MR to progress into severe form rapidly which will then result in left ventricular diastolic and systolic impairment.6 Leaflet motion of the valve is restricted particularly during diastole due to scarring and retraction of mitral valve leaflet and chordae [6]. The poor pumping action of the heart due to MS and MR in RHD will eventually lead to clinical heart failure.

Atrial fibrillation (AF) is most common arrhythmia associated with RHD and is known to correlate with left atrium size where the incidence of AF rises from 3\% when left atrial diameter is less than $40 \mathrm{~mm}$ and to $54 \%$ if more than $40 \mathrm{~mm}$ [7]. Left atrium enlargement is most common related to mitral valve disease where elevated left atrial pressure to create pressure gradient to assist diastole in MS and the blood leak from left ventricle to left atrium in MR. Valvular heart disease in RHD also results in fibrosis and degeneration of the atrial myocardium which causes disturbance impulse propagation in the atrial and eventually lead to AF [7]. Mitral valve which is calcified or prolapsing due to RHD can increase the occurrence of AF [7]. Patients with RHD which has AF and left atrial spontaneous echo contrast has increased risk of developing ischemic stroke [8]. Dysrhythmia occurs in AF causes contractile dysfunction, stasis and structural remodeling of the atrium which increases the risk of thromboembolism.7 In addition, AF causes stasis of blood which causes formation of thrombus or blood clot and eventually lead to embolism to the brain and results in ischemic stroke [8]. Oral anticoagulant or antiplatelet agents are required in the high-risk RHD to prevent stroke [9].
Pulmonary hypertension is a common complication of left heart disease which in this case due to RHD. Pulmonary hypertension occurs due to increased left ventricular or left atrial filling pressure in patients with RHD [10]. This will lead to increase in filling pressures in the left heart, which causes passive increase in backwards pressure on pulmonary veins and persistent increase in pulmonary venous pressure will eventually result in fragmentation of the alveolar-capillary walls [8]. Furthermore, persistent increase in pulmonary venous pressure and subsequent passive increase in pulmonary arterial pressure will lead to pathological changes in pulmonary veins and arteries such as muscularization and medial hypertrophy [10].

ARF can cause pancarditis but the ARF carditis mostly involved the endocardium which presents as vasculitis of the mitral valve [11]. Mitral valve that are scarred or damaged by RHD produces an alteration in the endothelial cells which in results increase the susceptibility for the colonization of the circulating bacteria on the surface of heart valve [11]. Survival of the bacteria that adhere on the surface of the heart valve will eventually leads to propagation of the infected vegetation [9]. Therefore, patients with RHD has a higher risk of developing infective endocarditis which is a bacterial infection of the heart valve.

\section{Management}

Oral penicillin is used to eliminate group A streptococcus from pharynx of patients with RHD but the benefits are largely based on anecdotal evidence [12]. However, penicillin can be used to clear group A streptococcal infection during ARF where benzathine penicillin G (benzylpenicillin) is given as a single intramuscular dose [13]. Antibiotic prophylaxis with benzathine penicillin $\mathrm{G}$ is recommended as a secondary prevention to prevent group A streptococcus infections in the future and to avoid ARF and development of RHD [13]. The required duration of secondary prevention for those with mild or no rheumatic heart disease is for a minimum of 10 years or until age 21 , for those with moderate heart disease is for until age 35 , and until age 40 or longer for those with severe heart disease [13]. Anti-inflammatory therapy such as corticosteroids treatment are recommended by some experts for patients with severe carditis although no evidence the therapy alters the long-term outcome of patients with ARF [2]. The risk of developing heart valve lesions after one year does not reduce with the use of corticosteroid [12]. Aspirin or nonsteroidal anti-inflammatory agents such as naproxen and high dose methylprednisone can be used to treat acute inflammatory process such as joint symptoms in patients with ARF [12].

Diuretics can be used judiciously in patients who develop pulmonary congestion [9]. Angiotensin-converting enzyme inhibitors can be used if there is symptomatic aortic regurgitation or left ventricular dysfunction [2]. Furthermore, drugs as such 
furosemide (frusemide), spironolactone, enalapril and digoxin can be used to manage RHD patients that develop heart failure.

Rheumatic chorea is usually self-limiting and resolves within weeks to months and therefore medications are not recommended due to potential serious adverse effects [2]. Valproic acid and carbamazepine are first-line treatment for chorea and are only used if the choreiform movement interfere with normal daily activities or place the patient at risk of injury [2].

Bed rest is also used to manage ARF but is not adequately study and physical activity should be restricted during ARF [12] Anticoagulants should probably be given to patient on bed rest and should be given to the patient who develops AF to prevent formation of thrombus [9].

Catheter-based intervention management such as percutaneous transvenous mitral commissurotomy (PTMC) can be used in patient with severe mitral stenosis and suitable valve morphology and the success of PTMC depends on the morphology of the mitral valve [14]. Open heart surgery is performed occasionally to manage more severe heart valve diseases that occur in patients with RHD [12]. Cardiac surgery is only performed until the acute inflammation has subsided, so that repair is easier and has a better long-term outcome [2]. However, surgical repair of mitral or aortic valve should be perform immediately if there is mitral or aortic regurgitation which occur due to rupture of chordae tendinae [2]. Surgical repair of damaged valve is preferred compared to rather than replacement to avoid complications such as thromboemboli and bleeding and longterm anticoagulant treatment $[2,12]$. However, valve replacement is the preferred surgical option if the patients develop severe heart valve lesions due to acute carditis and heart failure [12].

\section{Conclusion}

RHD is a preventable heart disease that has high occurrence among the developing and low-income countries. The most effective primary prevention method is antibiotic treatment of streptococcal throat infections [12]. Besides that, early intervention can increase the prognosis and outcome of the disease and prevent ARF from developing into RHD. Vaccines against $S$. pyogenes such as 26 valent type specific one is currently in phase II human trials while the $\mathrm{C}$ region $\mathrm{M}$ protein peptide vaccine, which is almost ready for trials in humans [12]. Prevention and control measures should be placed at greater emphasis to prevent RHD.

\section{Acknowledgement}

None

\section{Conflicts of Interest/Disclosures}

None

\section{References}

1. Gewitz Michael H, Baltimore Robert S, Tani Lloyd Y, Craig A Sable, Stanford T Shulman, et al. (2015) Revision of the Jones Criteria for the Diagnosis of Acute Rheumatic Fever in the Era of Doppler Echocardiography. Circulation 131: 1806-1818.

2. Sika-Paotonu D, Beaton A, Raghu A Andrew Steer, Jonathan Carapetis (2016) Acute Rheumatic Fever and Rheumatic Heart Disease. In Ferretti JJ, Stevens DL, Fischetti VA (Eds). Streptococcus pyogenes: Basic Biology to Clinical Manifestations, Oklahoma City (OK): University of Oklahoma Health Sciences Center.

3. Faé KC, Da Silva DD, Oshiro SE, Ana C Tanaka, Pablo M A Pomerantzeff, et al. (2006) Mimicry in Recognition of Cardiac Myosin Peptides by HeartIntralesional T Cell Clones from Rheumatic Heart Disease. The Journal of Immunology 176: 5662-5670.

4. Cunningham MW (2014) Rheumatic fever, autoimmunity, and molecular mimicry: the streptococcal connection. International reviews of immunology 33: 314-329.

5. Stanevicha V, Eglite J, Sochnevs A, Dace Gardovska, Dace Zavadska et al. (2003) HLA class II associations with rheumatic heart disease among clinically homogeneous patients in children in Latvia. Arthritis research \& therapy 5: R340-R346.

6. Mujib M, Desai RV, Ahmed MI, Jason L Guichard, Margaret A Feller, et al. (2012) Rheumatic heart disease and risk of incident heart failure among community-dwelling older adults: a prospective cohort study. Annals of medicine 44: 253-261.

7. Shankar PRB, Roa BH, Jaishankar S, M Narasimhan (2010) Current Perspectives: Rheumatic Atrial Fibrillation. Journal of atrial fibrillation 2: 222-222.

8. Gupta A, Bhatia R, Sharma G, Kameshwar Prasad, Mamta Bhushan Singh, et al. (2015) Predictors of Ischemic Stroke in Rheumatic Heart Disease. Journal of Stroke and Cerebrovascular Diseases 24: 2810-2815.

9. Rajamannan NM, Antonini-Canterin F, Moura L, José L Zamorano, Raphael A Rosenhek, et al. (2009) Medical therapy for rheumatic heart disease: is it time to be proactive rather than reactive? Indian heart journal 61: 14-23.

10. Guazzi, M, Galiè N (2012) Pulmonary hypertension in left heart disease. European Respiratory Review 21: 338-346.

11. Sullam PM, Drake TA, Sande MA (1985) Pathogenesis of endocarditis. The American Journal of Medicine 78: 110-115.

12. Cilliers AM (2006) Rheumatic fever and its management. BMJ (Clinical research ed.) 333: 1153-1156.

13. Ralph AP, Noonan S, Boardman C, Catherine Halkon, Bart I Currie, et al. (2017) Prescribing for people with acute rheumatic fever. Aust Prescr 40: 70-75.

14. Watkins DA, Beaton AZ, Carapetis JR, Ganesan Karthikeyan, Bongani M Mayosi, et al. (2018). Rheumatic Heart Disease Worldwide. Journal of the American College of Cardiology 72: 1397-1416. 
ISSN: 2574-1241

DOI: $10.26717 /$ BJSTR.2020.29.004793

Leong Tung Ong. Biomed J Sci \& Tech Res

(CC) (i) This work is licensed under Creative

Submission Link: https://biomedres.us/submit-manuscript.php

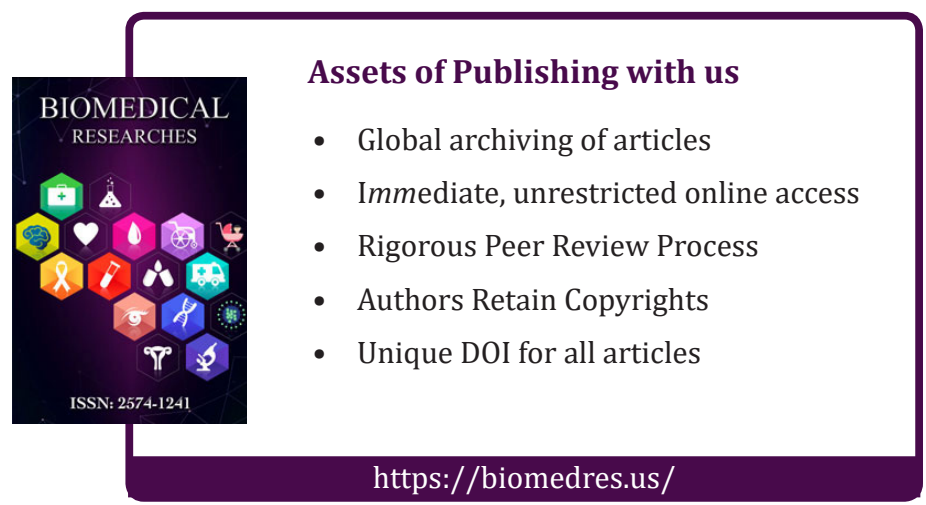

